\title{
Measurements of the top-quark mass using the ATLAS detector at the LHC
}

\author{
Michael Bender *i \\ Ludwig-Maximilians-Universität München \\ E-mail: mike.benderacern.ch
}

The latest measurements of the top-quark mass using the ATLAS experiment at the LHC are presented. A measurement based on a multidimensional template fit that can constrain the uncertainties on the energy measurements of jets is presented and combined with a measurement using dilepton events. Also measurements of the top-quark mass in the fully hadronic decay channel, as well as in t-channel enhanced topologies are shown. In addition, measurements are presented that use precision theoretical QCD calculations for both inclusive $t \bar{t}$ production and $t \bar{t}$ production with an additional jet to extract the top-quark mass in the pole mass scheme.

XXIV International Workshop on Deep-Inelastic Scattering and Related Subjects

11-15 April, 2016

DESY Hamburg, Germany

\footnotetext{
*Speaker.

On behalf of the ATLAS Collaboration
} 


\section{Top-quark production and decay}

The top quark, which was discovered in 1995 by the Tevatron experiments CDF and D0 [1, 2], is the heaviest known elementary particle. Since its discovery one major goal is to determine the top-quark mass $\left(m_{\text {top }}\right)$ precisely. With new experimental data from the LHC, the ATLAS [3] and CMS [4] Collaborations have managed to achieve a precision below $1 \mathrm{GeV}[5,6]$.

In proton-proton collisions at the LHC, top quarks are either produced in pairs through strong, or singly via electroweak interactions. Because of its short life time, the top quark decays before it hadronises and can therefore only be measured through its decay products. The branching ratios of the decay are proportional to the square of the corresponding CKM matrix element, which means that the top quark predominantly decays to a $\mathrm{W}$ boson and b quark. Since the $\mathrm{W}$ boson itself has a short lifetime, the detectable top-quark signature depends on the decay mode of the $\mathrm{W}$ boson.

In the case of $t \bar{t}$ production, one therefore distinguishes three decay channels. In the fully hadronic channel both $\mathrm{W}$ bosons decay hadronically, in the lepton + jets channel one $\mathrm{W}$ boson decays to leptons, the other to hadrons, and in the dileptonic channel both $\mathrm{W}$ bosons decay leptonically.

ATLAS has carried out measurements of the top-quark mass in all these channels, and in addition measured $m_{\text {top }}$ in a region enhanced with top quarks produced in the single top t-channel.

\section{Template measurements}

Most of the top-quark mass measurements done at ATLAS use the so called template method. In this method, Monte Carlo (MC) datasets for different top-quark masses are produced and the observables sensitive to the top-quark mass are parametrised as a function of $m_{\text {top }}$. The resulting template is then applied to the distributions measured in data and returns the measured top-quark mass. Therefore by determining $m_{\text {top }}$ with a template method, instead of measuring a mass in a defined scheme, the underlying top-quark mass parameter of the MC generator is measured. ${ }^{1}$

\subsection{Measuring $m_{\text {top }}$ in the fully hadronic channel}

In the fully hadronic decay channel, the final state comprises of at least six jets, two b-jets originating from the $t \bar{t}$ decay and four light quark jets from the hadronic W-boson decays. The main difficulty is therefore to differentiate between $t \bar{t}$ events and events originating from QCD multijet production. In order to reduce the overwhelming number of QCD events in the fully hadronic analysis [8], there are high jet $p_{\mathrm{T}}$ requirements (at least five jets with $p_{\mathrm{T}}>55 \mathrm{GeV}$ ) and a demand for exactly two b-tagged jets.

After all selection requirements, the final selection consists to about $28 \%$ of $t \bar{t}$ signal events, while the background is predominantly QCD multijet production. Since such high QCD jet multiplicities are not well modelled in MC simulations, the background is estimated from data with a crossmultiplication method. The number and shape of background events is extrapolated from orthogonal regions in phase space to the signal region. Those regions are defined by inverting the $\mathrm{b}$-tag and jet $p_{\mathrm{T}}$ requirements.

\footnotetext{
${ }^{1}$ At the moment, the difference between the MC mass definition and a well defined short-distance mass is estimated to be of the order of $1 \mathrm{GeV}[7]$.
} 


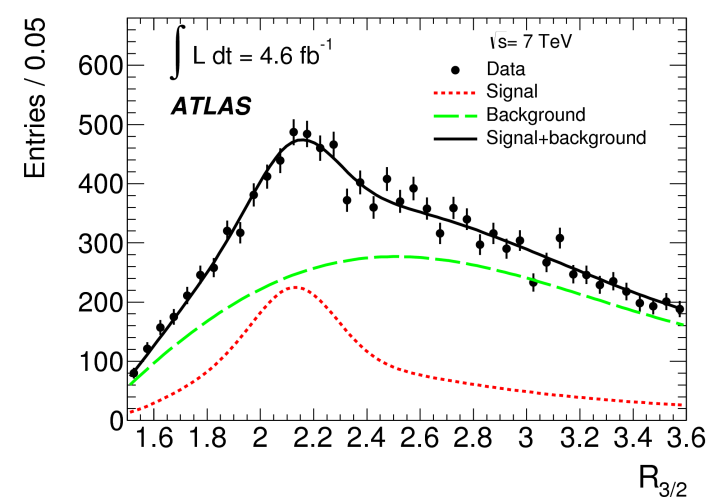

Figure 1: $\mathrm{R}_{32}$ as measured in data. The data is shown as black points, while the red and green line represent the fitted signal and background contributions, respectively. [8]

Figure 1 shows the observable used to determine $m_{\text {top }} . R_{32}$ is defined as the ratio of the reconstructed top-quark mass $\left(m_{\mathrm{jjj}}\right)$ and the corresponding reconstructed W-boson mass $\left(m_{\mathrm{jj}}\right)$, both of which are determined via a kinematic likelihood fit. The advantage of using $\mathrm{R}_{32}$ instead of $m_{\mathrm{jjj}}$ is that overall systematics which $m_{\mathrm{jjj}}$ and $m_{\mathrm{jj}}$ have in common cancel each other, therefore resulting in a lower overall uncertainty of the measurement. The estimated top-quark mass is $m_{\text {top }}=175.1 \pm 1.4$ (stat) \pm 1.2 (syst) GeV.

\subsection{Measuring $m_{\mathrm{top}}$ in the lepton + jets and dileptonic channel}

ATLAS has performed a combination of its $m_{\text {top }}$ measurements in the lepton + jets and dilepton channels [5]. The analyses are selecting events with at least one b-tagged jet, one (two) charged lepton(s) and missing transverse energy.

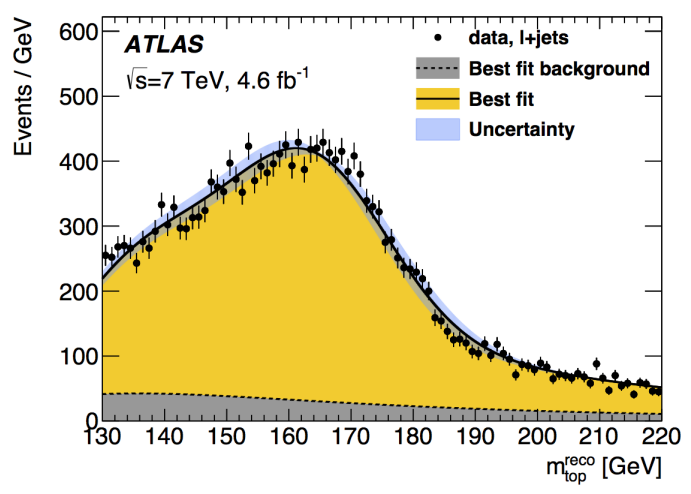

(a)

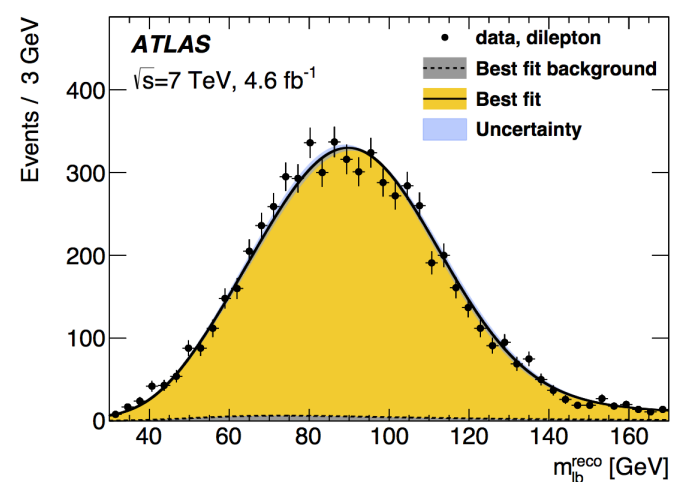

(b)

Figure 2: Fitted distributions of (a) $m_{\mathrm{jjj}}$ and (b) $m_{\mathrm{lb}}$. Black points represent the data, while the fitted signal and background contributions are shown in yellow and grey, respectively. [5]

In the case of the lepton + jets analysis $m_{\mathrm{jjj}}$ is chosen as observable for $m_{\mathrm{top}}$ (shown in Fig. 2a). To reduce the influence of the jet energy scale, $m_{\mathrm{jj}}$ and a quantity called $\mathrm{R}_{\mathrm{bq}}$ are considered in the extraction of $m_{\mathrm{top}}$, as well. $\mathrm{R}_{\mathrm{bq}}$ is defined as the ratio of the scalar sum of the b-tagged jet's transverse momenta and the transverse momenta of the jets reconstructed as the hadronically decaying $\mathrm{W}$ boson. By performing a three dimensional template fit, the jet scale factors are measured in situ, 
thereby reducing the overall uncertainty on the top-quark mass. In this channel the mass of the top quark is measured to be $m_{\text {top }}=173.3 \pm 0.8$ (stat $+\mathrm{JSF}+\mathrm{bJSF}$ ) \pm 1.0 (syst) $\mathrm{GeV}$.

In the dilepton measurement, $m_{\text {top }}$ is determined from the invariant mass of the lepton and b-tagged jet system $\left(m_{\mathrm{lb}}\right)$. Figure $2 \mathrm{~b}$ shows the template fit to the $m_{\mathrm{lb}}$ distribution, which yields a value of $m_{\text {top }}=173.8 \pm 0.5$ (stat) \pm 1.3 (syst) GeV.

Since both analyses are orthogonal to each other, one can increase the sensitivity and therefore decrease the overall uncertainty by combining the results from both channels. The combination takes into account the correlation between the different systematic uncertainties and results in a top-quark mass estimate of $m_{\text {top }}=173.0 \pm 0.5$ (stat) \pm 0.8 (syst) GeV. This corresponds to a gain in precision of $28 \%$ compared to the single lepton + jets measurement.

\subsection{Measuring $m_{\text {top }}$ in the single top t-channel}

As explained, top quarks can also be produced in electroweak interactions. The analysis in topologies enhanced with t-channel top quarks [9] aims to measure $m_{\text {top }}$ in exactly such a process.

The measurement is designed to select top quarks in which the corresponding $\mathrm{W}$ boson decayed leptonically, therefore asking for exactly one charged lepton and missing transverse energy. In contrast to $t \bar{t}$ measurements, the final state consists only of the decay products of a single top quark, accompanied by an additional jet. The main challenge therefore is to separate the signal from $\mathrm{W}+\mathrm{jets}$ background events, which have the larger cross section. To achieve this, a neural networkbased discriminant is used for the event selection, shown in Fig. 3a. By requiring a value larger than 0.75 , most of the background events are discarded. After all requirements, the final selection contains mainly t-channel top-quark events (about 46\%) while the background contribution is only about $28 \%$. The remaining $26 \%$ are mostly $\mathrm{t} \overline{\mathrm{t}}$ events and other single top processes, which are also considered as signal events.

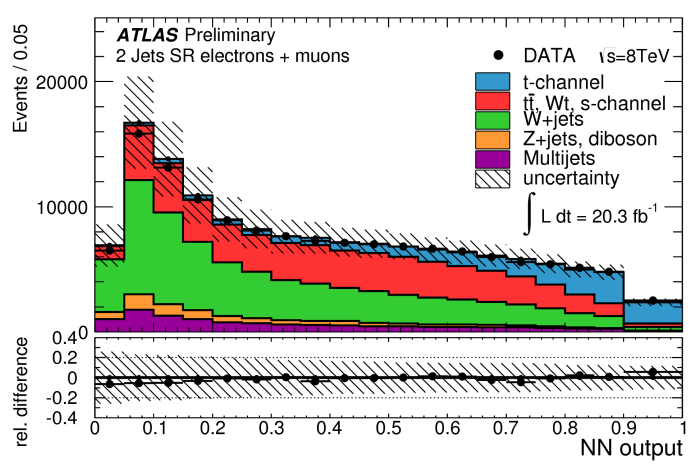

(a)

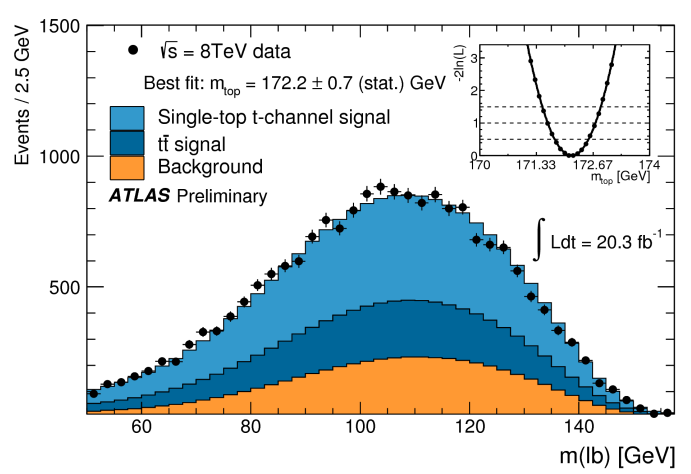

(b)

Figure 3: The output of the neural network for different signal and background processes is shown in (a). Figure (b) presents the $m_{\mathrm{lb}}$ distribution after the final selection, data are shown in black, estimated signal and background contributions, in blue and orange, respectively. [9]

As in the dilepton analysis, the $m_{\mathrm{lb}}$ observable (shown in Fig. $3 \mathrm{~b}$ ) is used to determine $m_{\text {top }}$. A template fit to the $m_{\mathrm{lb}}$ distribution yields a result of $m_{\mathrm{top}}=172.2 \pm 0.7$ (stat) \pm 2.0 (syst) GeV. This result represents the first measurement of $m_{\text {top }}$ in a phase space dominated by electroweakly produced top quarks, and is consistent with other analyses performed in tt final states [5, 8]. 


\section{Cross section measurements}

Cross section mass measurements exploit the relation between the mass of a particle and its production cross section. By measuring the (differential) cross section of top-quark production one can derive $m_{\text {top }}$ in a theoretically well defined way. It is the big advantage of such measurements compared to template methods, that instead of the MC top mass parameter, the top-quark pole mass $\left(m_{\text {top }}^{\text {pole }}\right)$ is measured. ${ }^{2}$

\subsection{Measuring $m_{\text {top }}$ from the $\mathbf{t} \overline{\mathbf{t}}$ cross section}

ATLAS has presented a combined measurement of the tt production cross section in $\sqrt{s}=7 \mathrm{TeV}$ and $8 \mathrm{TeV}$ data [10]. For this measurement, dileptonic $\mathrm{t} \overline{\mathrm{t}}$ events have been selected by requiring an oppositely charged electron-muon pair in the final state. This allows for a very clean dataset, in which background contributions are down to a percent level.

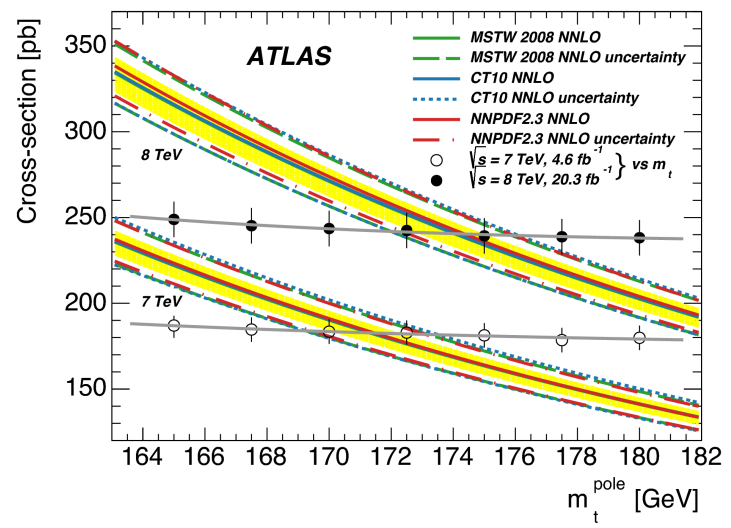

Figure 4: Measured cross sections for $\sqrt{s}=7 \mathrm{TeV}$ and $8 \mathrm{TeV}$ compared to theory prediction. [10]

By using the theoretically predicted dependence of the cross section on the top-quark mass, $m_{\text {top }}$ can be inferred from the measured $\mathrm{t} \overline{\mathrm{t}}$ cross sections at $\sqrt{s}=7 \mathrm{TeV}$ and $8 \mathrm{TeV}$. A combination of both results yields an estimate for the top-quark mass of $m_{\mathrm{top}}^{\text {pole }}=172.9_{-2.6}^{+2.5} \mathrm{GeV}$, which is in good agreement with the direct measurements $[5,8,9]$.

\subsection{Measuring $m_{\text {top }}$ with the differential $\mathbf{t} \overline{\mathbf{t}}+1$-jet cross section}

In addition to the inclusive $t \bar{t}$ cross section mass determination, ATLAS has also measured the

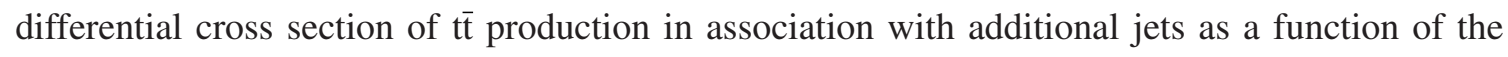
invariant mass of the $t \bar{t}+1$ jet system [11]. It has been shown, that this alternative approach of a cross-section based mass measurement is very sensitive to the top-quark pole mass [12].

In the analysis, $t \bar{t}$ events from the lepton + jets decay have been selected, therefore requiring exactly one charged lepton, missing transverse energy and due to the demand for an additional jet, at least five jets with $p_{\mathrm{T}}>25 \mathrm{GeV}$. In order to be able to compare the experimental data to the theory prediction, the measured distribution was corrected for hadronisation and detector effects. The top-quark pole mass is then derived by fitting the theory prediction for different top-quark masses to the corrected distribution. The top-quark pole mass has been measured to be $m_{\mathrm{top}}^{\text {pole }}=173.7 \pm$ 1.5 (stat) \pm 1.4 (syst) ${ }_{-0.5}^{+1.0}$ (theo) $\mathrm{GeV}$, which also agrees well with direct measurements $[5,8,9]$.

\footnotetext{
${ }^{2}$ In principle, one could also use other mass schemes, but $m_{\text {top }}^{\text {pole }}$ has been chosen for the ATLAS analyses.
} 


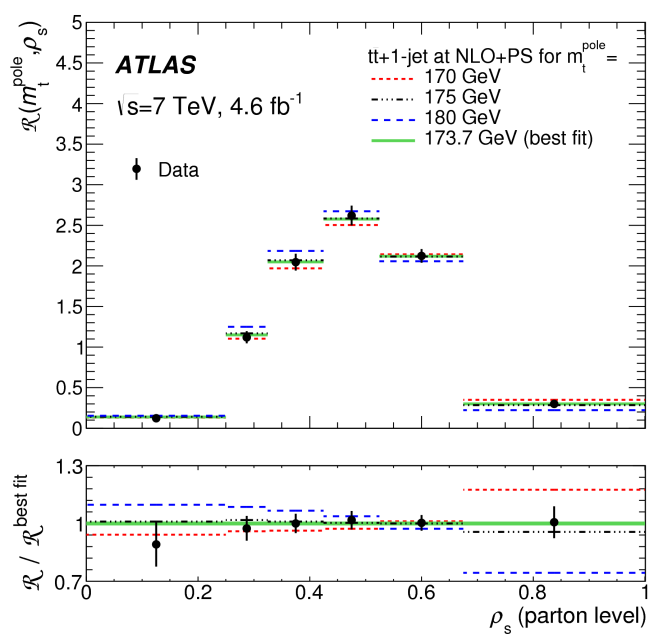

Figure 5: Signal distribution as function of the inverse of the $t \bar{t}+1$ jet system. Corrected data is shown in black, the theory prediction for different top-quark masses is also indicated. [11]

\subsection{Conclusion}

ATLAS has performed several top-quark mass measurements with different methods in different decay topologies. All results are in good agreement with each other, and also agree well with the current world average value $m_{\text {top }}=173.3 \pm 0.3$ (stat) \pm 0.7 (syst) $\mathrm{GeV}$ [13].

With the enhanced cross sections at $\sqrt{s}=13$ and $14 \mathrm{TeV}$ and increased statistics of future LHC data taking, an unprecedented amount of data will become available for further enhancing the precision of upcoming $m_{\text {top }}$ measurements.

\section{References}

[1] F. Abe et al. (CDF Collaboration), Phys. Rev. Lett. 742626 (1995), arXiv:hep-ex/9503002

[2] S. Abachi et al. (D0 Collaboration), Phys. Rev. Lett. 742632 (1995), arXiv:hep-ex/9503003

[3] ATLAS Collaboration, JINST 3 S08003 (2008)

[4] CMS Collaboration, JINST 3 S08004 (2008)

[5] ATLAS Collaboration, Eur. Phys. J. C 75:330 (2015), arXiv:1503.05427 [hep-ex]

[6] CMS Collaboration, Phys.Rev. D 93072004 (2016), arXiv:1509.04044 [hep-ex]

[7] S. Moch et al., DESY-14-081, MITP-14-036, arXiv:1405.4781 [hep-ph]

[8] ATLAS Collaboration, Eur. Phys. J. C 75:158 (2015), arXiv:1409.0832 [hep-ex]

[9] ATLAS Collaboration, ATLAS-CONF-2014-055, CDS record 1951323

[10] ATLAS Collaboration, Eur. Phys. J. C 74:3109 (2015), arXiv:1406.5375 [hep-ex]

[11] ATLAS Collaboration, JHEP10(2015)121, arXiv:1507.01769 [hep-ex]

[12] S. Alioli et al., Eur. Phys. J. C 73:2438 (2013), arXiv:1303.6415 [hep-ph]

[13] ATLAS, CDF, CMS and D0 Collaborations, arXiv:1403.4427 [hep-ex] 